\title{
ABHÄNGIGE INSZENIERUNGEN DER UNABHÄNGIGKEIT. DER INDEPENDENT-DISKURS IN MUSIKZEITSCHRIFTEN
}

\section{André Doehring}

Wer oder was im Musikbetrieb als »Independent « bezeichnet wird, ist genauso wenig klar, wie die jeweils so benannten Klangstrukturen kongruent sind. Dennoch resp. gerade deshalb eignet sich das ursprünglich englische Adjektiv independent, mitunter zum Diminutiv »Indie « substantivierend verhätschelt, als Ordnungsbegriff populärer Musik: ${ }^{1}$ Es fasst das zunächst Unzusammenhängende zu einer Kategorie zusammen, die nun Genres (die meist als Komposita auftreten: Indie-Electronica, Independent Rock usw.), Bands, Interpreten, Plattenfirmen, Magazine, Filme und vieles mehr umfasst, und signalisiert, dass die (auch: musikalische) Welt scheinbar einfach in Abhängiges und Unabhängiges geteilt werden könne.

Tatsächlich müssen Musikbegriffe jedoch stets als diskursive Begriffe gelesen werden. Das bedeutet erstens, dass sie Klanggebilde innerhalb eines historisch gewachsenen Raumes bezeichnen, in dem nur das gesagt und gedacht werden darf bzw. kann, was sich der Ordnung des Diskurses fügt. Erst durch den Akt der Benennung gemäß den herrschenden Sprachregelungen können diese Klanggebilde nun als Musik in den Diskurs eintreten, d.h. dort wahrgenommen werden und ihre Wirksamkeit für die Teilnehmer entfalten (vgl. Wicke 2004). Hier fungieren sie nun als »Klassifikationsprinzipien«, da sie reale Klangereignisse bändigen und Zufälligkeiten eliminieren (vgl. Foucault 1991: 17). Zweitens stützen sich Musikbegriffe wie alle diskursiven Aussagen auf eine institutionelle Basis, ein mitunter übersehener Aspekt der Diskurstheorie, der allerdings von musiksoziologischer Relevanz ist: Institu-

1 Die Oxford Dictionaries (2012) beziehen »indie« - im Unterschied zu »independent « - konkret auf »a pop group, record label, or film company«; es kann im Englischen sowohl als Adjektiv wie auch als Nomen gebraucht werden. Diese Verwendung hat sich auch im Deutschen eingebürgert (wenngleich der Duden nur das Hauptwort kennen mag), daher wird im Text sowohl von indie als auch von Indie gesprochen werden, immer wieder unterbrochen von der Verwendung von independent bzw. Independent als nachdrückliche Erinnerung an die ursprüngliche Bedeutung als Unabhängigkeit von etwas oder jemandem. 
tionen als habitualisierte Formen des Handelns und der sozialen Interaktion erhalten ihren Sinn wie auch ihre Rechtfertigung durch die sie umgebende Kultur und tragen zum Erhalt dieser Kultur bei, solange sie ihre handlungsleitende Macht aufrechterhalten können (vgl. Gukenbiehl 2000: 142). Für Foucault (1991: 15) ist die institutionelle Basis ein »Geflecht von Praktiken «, das in der Pädagogik einerseits und andererseits dem »System der Bücher, der Verlage und der Bibliotheken « - oder für den hiesigen Zusammenhang kürzer und umfassender: in den Medien - (re)produziert wird. Sie ist verantwortlich für die »Art und Weise, in der das Wissen in einer Gesellschaft eingesetzt wird, in der es gewertet und sortiert, verteilt und zugewiesen wird« (ebd.). Hinzuzufügen ist, dass neben den Prozessen der Wertung auch Verwertungsprozesse für die Verteilung des Wissens um die Begriffe sorgen und somit zur Aufrechterhaltung der institutionellen Basis beitragen. Für eine wissenschaftliche Auseinandersetzung mit dem Begriff Independent ist daher eine Genealogie - im Sinne Foucaults die Analyse der Normen, Erscheinungs-, Wachstums- und Veränderungsbedingungen (ebd.: 39) - nötig, die auch auf die institutionelle Basis blickt.

Denn eine bloß musikimmanente Vorgehensweise bei der Beschäftigung mit so unterschiedlich klingender Musik wie beispielsweise von Black Flag (Bannister 2006: 93ff.), den Artic Monkeys (Anon. 2012) oder Dntel (Wiene 2012: 89) kann nicht erklären, warum - um im Beispiel zu bleiben - ein Wissenschaftler (Bannister), ein virtuelles Aggregat von Menschen in Wikipedia und ein Musikjournalist (Wiene) die jeweiligen Klänge als independent bzw. Indie bezeichnen. Independent, so wird im Weiteren ausgeführt, ist als ein Diskurs über populäre Musik zu verstehen, der spezielle Wertmuster ausgebildet hat, die einer Klangstruktur zugesprochen werden können, um sie im Musikprozess von Herstellung über Vertrieb und Verkauf bis zur Rezeption strategisch zu positionieren. Diese Wertmuster werden in der musikalischen Praxis, verstanden im umfassenden Sinne Kurt Blaukopfs (1984: 20f., $2010)^{2}$, durch Medien wie Musikzeitschriften den Diskursteilnehmern verfügbar gemacht. Musikzeitschriften sind Institutionen, die einer Diskursgemeinschaft Begriffe wie Independent liefern, erläutern und pflegen. Diese Institutionen haben eigene Interessen, sie sind in einem $\mathrm{zu}$ bestimmenden musikjournalistischen Feld mit diversen anderen Akteuren und Institutionen verflochten und besitzen zu spezifizierende Organisations- wie Produktions-

2 In Musik im Wandel der Gesellschaft definiert Blaukopf (1984: 21) den Begriff der musikalischen Praxis als »alle Handlungen und Unterlassungen im musikalischen Bereich « sowie auch die »theoretische Reflexion über diese musikalische Praxis selbst, das heißt das Denken über Musik, welches auf der jeweiligen Praxis beruht und diese zu steuern vermag.« 
verhältnisse. Nicht zuletzt sind ihre Medienakteure Teil der Institution Musikzeitschrift ${ }^{3}$ und tragen zur medialen Belebung des Begriffs Independent durch die monatliche (Re-)Produktion die Wertmuster bei, die uns als Indie gegenübertreten. Das heißt, diese Musikkommunikatoren inszenieren das Klingende auf eine Weise, die fachlichen Anforderungen und diskursiven Ansprüchen genüge tut, ohne dass die Inszenierenden, die Musikjournalisten selber, diese Mächte als ihr Handeln bestimmend wahrnehmen müssen.

\section{Zum Begriff der Inszenierung}

In den Kulturwissenschaften wird seit den 1980er Jahren in zunehmendem Ausmaß von einem Theatralitätsmodell von Kultur gesprochen (vgl. Warstat 2005: 358). In aller Kürze und möglichst einfach gefasst, verbindet sich damit die Vorstellung, dass wir ähnlich wie im Theater durch die Beobachtung anderer Akteure etwas über die Welt erfahren, die dargestellt wird, der wir aber zugleich auch angehören und somit selber Darsteller in diesem Theater sind. Das Paradigma vom kulturellen Leben als Aufführung, wo etwas oder jemand bewusst exponiert oder angeschaut wird (vgl. ebd.), hat etwa bei Erving Goffman (1983) zur anthropologischen Feststellung geführt, wir alle spielten Theater, wenn wir uns darstellen. Der Soziologe Herbert Willems hat die Idee theatraler Kultur auf die Medien erweitert:

»In der heutigen Gesellschaft spielen die Massenmedien die wichtigste erfahrungsgenerative Rolle. [...] In evolutionärer Kontinuität und funktionaler Überbietung des Theaters und anderer kosmologischer Institutionen (etwa religiöser Art) haben sich die Massenmedien fraglos zum gesellschaftlichen Theatralitäts- und Realitätszentrum entwickelt« (Willems 1998: 64).

Insofern wäre es also nur konsequent, die mittlerweile in den Kulturwissenschaften »allgegenwärtigen« (Fischer-Lichte 2005a: 149) Gebräuche von Vokabeln der Theaterwelt wie etwa Theatralität, Performance, Aufführung oder hier: Inszenierung auf die Vorgänge in und um musikbezogene Massenmedien anzuwenden - wenn denn (eine wenigstens fachinterne) Klarheit herrschte, was tatsächlich sich hinter diesen Begriffen verbirgt. Die Musikwissenschaft scheint, so viel muss gesagt sein, wieder einmal eine Debatte um kulturwissenschaftliche Leitbegriffe - freundlich ausgedrückt - umse-

3 Dass hier tatsächlich Menschen unter selbst gewählten, aber nicht geschaffenen Verhältnissen erheblich und oft gerne und unterbezahlt zur symbolischen Aufladung der Musikwaren beitragen, ist in der bisherigen Forschung über Musikzeitschriften so gut wie nie thematisiert worden, was ich an anderer Stelle ausführlicher kritisiere (Doehring 2011: 24ff. u. 285ff.). 
gelt, in Wahrheit aber wohl eher verschlafen zu haben. So weist Uwe Wirth (2002: 9) darauf hin, dass bspw. unter dem Begriff >Performanz < keineswegs jede Disziplin dasselbe fasse; in seiner Aufzählung von fachinternen Beispielen fehlt die Musikwissenschaft. Und selbst im Jahr 2011 kann ein interdisziplinärer Sammelband zu einer »Zwischenbilanz« der Performativitätsforschung, wie die Herausgeber im Vorwort schreiben (Hempfer/Volbers 2011: 8), ohne einen Beitrag aus der Musikwissenschaft mühelos gefüllt werden. ${ }^{4}$ Daher scheint es im Folgenden angeraten, den Begriff der Inszenierung, mit dem hier gearbeitet werden soll, kurz theoretisch einzuführen. Meinem Verständnis zufolge haben wir es vor allem mit zwei Disziplinen zu tun, die für eine gewinnbringende Übertragung in den hiesigen Zusammenhang zu inspizieren sind: die Theaterwissenschaft und die Soziologie.

Unter Inszenierung wird in der Theaterwissenschaft der »Vorgang der Planung, Erprobung und Festlegung von Strategien verstanden, nach denen die Materialität einer Aufführung performativ hervorgebracht werden soll« (Fischer-Lichte 2005a: 146). Derart werden Ereignisse als gegenwärtig wahrgenommen, sie treten in Erscheinung und konstituieren Wirklichkeit. Gleichzeitig eröffnen Inszenierungen aber auch Spielräume, die nicht-geplante und nicht-inszenierte Handlungen, Verhaltensweisen und Ereignisse ermöglichen (vgl. ebd.).

Damit sind Inszenierungen schöpferische Prozesse, in denen »in spezifischer Weise Imaginäres, Fiktives und Reales (Empirisches)« (Fischer-Lichte 1998: 88) hervorgebracht wird. Inszenieren ist der kreative und transformierende Umgang des Menschen mit sich selbst und seiner Umwelt (vgl. ebd.), ein Vorgang, der »durch eine spezifische Auswahl, Organisation und Strukturierung von Materialien/Personen etwas zur Erscheinung bringt « - und dieses Etwas kann als der Inszenierung »Vorausliegendes« erst durch sie hervorgebracht werden (ebd.: 87). Fischer-Lichte erlaubt somit, den Begriff in eine konstruktivistische Lesart zu überführen: die Welt ist, welches Bild wir uns von ihr machen - sollen, dürfen und können. Für die Kulturwissenschaften, unter die ich die Musikwissenschaft subsumiere, ist der Begriff deshalb so reizvoll, weil wir eine Kultur der Inszenierung ebenso erkennen und beschreiben können wie die Inszenierung von Kultur.

4 Knappe zehn Jahre, nachdem Wirth (2002: 10) dem Begriff Performanz »Hochkonjunktur « attestierte, dreizehn Jahre nach dem Erscheinen des Sammelbands zur Inszenierungsgesellschaft (Willems/Jurga 1998b), scheinen im Jahr 2011 diese Begriffe in der deutschsprachigen musikologischen Disziplin auf höhere Resonanz zu stoßen, wenn die hohe Beteiligung an der ASPM-Jahrestagung »Populäre Inszenierungen - Inszenierungen des Populären« in Paderborn als Indikator genommen werden darf. 
In der Soziologie (Willems/Jurga 1998a: 11) ist der Begriff der Theatralität als umfassendes, »totales Meta-Konzept « dargestellt worden, das jegliche Art von »(Re-)Präsentation « im Sinne einer Inszenierung umfasst. Willems (1998) erweitert den Begriff um mikro- wie makrosoziologische und vor allem machtanalytische Komponenten. So führt er Norbert Elias' Begriff der Figuration ein, da in der Analyse von Inszenierungen sonst »relevante Strukturbedingungen, Sinnbezüge, Beziehungsnetze und Handlungsketten jenseits der Interaktion und ihrer situativen Lokalität« (Willems 1998: 50) aus dem Blick geraten. Man müsse den »komplexen strukturellen und prozessuellen [sic] Gesamtzusammenhang der Praxis, ihre Gefüge und ihre >Fügungen « untersuchen (ebd.: 51), um einzelne Inszenierungen verstehen zu können. ${ }^{5}$ Durch die Integration von Bourdieus Habitustheorie zeigt er die Sedimentierung von gesellschaftlich erzeugter Praxis, die in den Subjekten wirksam wird, wenn sie Theater spielen, wie sie auch im Zuschauer und in den Inszenierenden wiederum als rahmende Instanz Anwendung findet. Denn die »Erscheinungsformen und Sinnvoraussetzungen von Theatralität [sind] durch und durch historisch« (ebd.; Hervorhebung im Original).

Zusammengefasst folgere ich für die Beschäftigung mit musikbezogenen Medien daraus, dass auch mediale Inszenierungen strategische, geplante, gemeinsame Handlungen sind, die in der Aufführung (der Veröffentlichung) etwas hervorbringen, was wohl vorher existierte, aber erst durch die Inszenierung für ihre Teilnehmer fassbar wird. Inszenierungen sind somit performative Prozesse, die innerhalb eines materialen, personalen und öffentlichen Rahmens stattfinden, der als gesellschaftliche Praxis von Machtansprüchen durchzogen ist. Inszenierungen zu verstehen bedeutet, die zum Theater und zur Inszenierung gehörenden Strukturen ebenso in den Blick zu bekommen wie die Akteure, die auf, vor und hinter der Bühne Teil der Inszenierung sind. In meiner Arbeit über Popmusikjournalismus und -journalisten schlage ich dafür die Verwendung von Bourdieus Begriffen des Feldes und des Habitus vor (Doehring 2011: 49ff.). Die Strukturen und Regeln des Feldes Popmusikjournalismus, auf dem die Musikjournalisten agieren, sind gleichermaßen Teil der Untersuchung von medialen Inszenierungen wie die Akteure und ihre Habitus. Juristische, ökonomische, politische, technologische, organisatorische und ideologische Aspekte ermöglichen und bedingen das Handeln der Medienakteure, deren Habitus ebenfalls prägend in mediale Inszenierungen eingehen. Selbstverständlich und holistisch korrekt gehören weiterhin die Ebene der Medieninhalte wie auch die

5 Fischer-Lichte (2005b: 235) weist für performative Äußerungen ebenfalls darauf hin, dass es Gelingensbedingungen gebe, die vor allem institutioneller und sozialer Natur seien. 
Rezeption dieser Medien dazu. Allein: Beide waren bisher auf der Agenda, wenn es um die wissenschaftliche Beschäftigung mit Musikzeitschriften ging. Es gilt, die materiellen und habituellen Produktionsverhältnisse, die der Erstellung von Inhalten und deren Rezeption vorgängig sind, in den Fokus der Wissenschaft zu bekommen.

\section{Zum Begriff Independent}

Der Begriff wurde in Bezug auf populäre Musik erstmals auf US-amerikanische Labels der 1930er Jahre wie OKeh Records, Black Swan, Gennett u.a. angewandt (Wicke/Ziegenrücker/Ziegenrücker 2007: 338). In seiner dann folgenden Verwendung als Organisationsbegriff populärer Musikpraxis ist indes keine Einheitlichkeit zu beobachten. ${ }^{6}$ Für eine ausführliche historische Darstellung ist hier nicht der geeignete Ort, daher beschränke ich mich auf eine möglichst systematisierende Vorstellung von typischen Aspekten. Wenn von Independent gesprochen und geschrieben wird, ist typischerweise einer oder sind mehrere der folgenden vorgeschlagenen Dimensionen des Begriffs betroffen:

1. Musikindustrielle Aspekte, die ökonomische und organisatorische Aspekte des Besitzes, Vertriebs und Verkaufs von Musik fassen.

2. Ästhetische Aspekte, die sich auf musikalische Entscheidungen, Praxen und Theorien erstrecken.

3. Soziale Aspekte, die sich auf Verhalten, Integrations- wie Differenzbewegungen beziehen.

4. Politisch-ethische Aspekte, die sich als Haltung (state of mind) zu erkennen geben.

Als Independent-Band könnte bspw. eine mit zwei Gitarren, Bass und Schlagzeug sowie Gesang besetzte Band zum überwiegenden Teil männlichen Geschlechts ${ }^{7}$ gelten, die das Ausstellen virtuoser technischer Fähigkeiten an ihren Instrumenten bewusst vermeidet; die als >künstlerisch ambitioniert gelten, da sie das Startum ablehnen und kommerziellen Erfolg nicht anzustreben scheinen; die ihre Alben selbst oder über ein inhabergeführtes Label und einen Vertrieb, der nicht im Besitz eines der verblie-

6 Vgl. bspw. Alsmann 1985; Gruber 1995; Büsser 1997; Azzerad 2002; Broven 2009; Oakes 2009; Eisewicht/Grenz 2010; Moore 2010.

7 Trotz mitunter offensiv geäußerter feministischer Haltungen ist die Welt des Independent männlich dominiert (vgl. bspw. Clawson 1999; Davis 2001; Bannister 2006). 
benen $\mathrm{drei}^{8}$ marktbeherrschenden Medienunternehmen ist, herstellen, lizensieren und vertreiben. Die Band könnte weiterhin ihr Bemühen zur Reduzierung der Emission klimaschädlicher Gase kommunizieren, woraufhin ihre Fans Fahrgemeinschaften zu Konzerten der Band bilden. So geschehen bei Radiohead, einer Band, die zwar ihr Klangspektrum durch Hinzunahme elektronischer Instrumente erweitert hat, aber ihre Alben mittlerweile selbst vertreibt, ${ }^{9}$ sich (auch klima-)politisch äußert und daher in der Musikpresse als Idealtypus einer Indie-Band gilt. ${ }^{10}$

In ihrer empirischen Arbeit über die Independent-Szene Großbritanniens überlegt Wendy Fonarow (2006: 28ff.), die heute als »Indie Professor « für den englischen Guardian eine Kolumne füllt, ${ }^{11}$ woher diese auch bei anderen Bands beobachtbaren Zuschreibungen (bspw. in Azzerad 2002) stammen. Sie erkennt hier ein zugrundeliegendes Wertmuster, das sie als Erbe von Puritanismus und Romantizismus interpretiert. Independent vertritt demzufolge Werte wie erstens Purismus und moralischen Rigorismus, die sich als Askese äußern können. Zweitens betont Independent Autonomie, einen Wert, der auf der puritanischen Absonderung der Gemeinde von der päpstlichen Autorität basiert, und hegt drittens Präferenzen für das Natürliche, was die Ablehnung von Technologie einbegreifen und sich in eine nostalgische Verklärung des Früher wenden kann. Außerdem verlangt Independent von den Diskursteilnehmern Leidenschaft und Kreativität, wobei besonders die Ausführenden im romantischen Genieglauben überhöht werden. Dieses Wertmuster ist auf jeder der oben vorgeschlagenen Ebenen zu beobachten.

8 Zum Zeitpunkt der Abfassung dieses Aufsatzes veröffentlicht der EU-Wettbewerbskommissar Joaquin Alumnia die Zustimmung der EU-Kommission zur Zerschlagung des britischen Musikkonzerns EMI an Sony und Universal. EMI war nach dem Kauf durch die Kapitalbeteiligungsgesellschaft Terra Capital des britischen Investors Guy Hands 2007 in finanzielle Nöte geraten, Anfang 2011 wurde sie vom US-amerikanischen Finanzdienstleister Citibank gepfändet. Im April 2012 genehmigte die EU-Kommission bereits die Übernahme des Verlagsgeschäfts der EMI durch eine von Sony angeführte Investorengruppe. Nun steht nur noch die Zustimmung der US-amerikanischen Kartellbehörden zur Übernahme des Tonträgergeschäfts durch Universal aus, womit sich dann drei Unternehmen den Markt teilen würden (vgl. Oldag 2012).

9 Bekanntermaßen wurde ihr Album In Rainbows (2007) zunächst über ihre Website vertrieben, wo die User sich überlegen durften, ob und falls ja, welchen Preis sie für die Musik bezahlen wollten. Das Anbieten eines potentiell kostenlosen Downloads scheint das kommerzielle Desinteresse der Band zu illustrieren - die aber zwei Monate später die Musik auf den üblichen Wegen allein käuflich verfügbar machte.

10 Vgl. die Aussagen der Popmusikredakteure in Doehring 2011: 188.

11 Vgl. http://www.guardian.co.uk/music/series/ask-the-indie-professor (Zugriff: 26.9.2012). 
Independent kann demnach als Musikkonzept verstanden werden, in dem Wert- und somit Sinnmuster für alle am Musikprozess Beteiligten gefasst sind - ohne allerdings für alle Gleiches zu bedeuten. Denn als diskursiver Begriff, so bezeichnet inn auch Fonarow (2006: 26), ist Independent durch und durch historisch, kontingent und umkämpft. Sind mit Independent also beim ersten Auftauchen des Begriffs die kleinen, nicht marktbeherrschenden Tonträgerunternehmen gemeint, wurde mit der einsetzenden Printveröffentlichung der ersten »Independent Charts« ab 1979/80 Independent anhand der Form des Vertriebs festgemacht: Nicht mehr die Besitzverhältnisse des Labels, sondern die des Vertriebs und seine Form waren ausschlaggebend - was den Major Labels die Möglichkeit bot, nun auch diese Charts zu dominieren. ${ }^{12}$ In den 1970er Jahren hatten sich in Großbritannien und Nordamerika, aber auch in der Bundesrepublik ${ }^{13}$ zunehmend von Musikern betriebene Labels gebildet, die als Institutionen einer sich entwickelnden Szene die Mitglieder mit visuellen wie auditiven Inhalten und vor allem Konzerterlebnissen versorgten, zu einer Zeit, in der die großen Plattenfirmen noch nicht auf die Marktfähigkeit dieser Nischenmusik vertrauten. Um Labels wie SST, von Black Flag-Gitarrist Greg Ginn Ende 1978 gegründet (Azzerad 2002: 18), gruppierten sich Bands wie Black Flag, The Minutemen, Hüsker Dü, Meat Puppets, Sonic Youth oder Dinosaur Jr., deren politische und ethische Haltung sowie ungeheure Arbeitsmoral sich in einer hohen Veröffentlichungszahl und fast ununterbrochenen selbstorganisierten Tourneen manifestierten und somit verbreiteten. Diese Bands, ihre Musik wie ihre Haltung, gelten - nicht zuletzt durch wirkungsmächtige Heroengeschichten des »Indie Underground « wie die von Azzerad (2002) - als Prototypen wie auch Elemente eines Kanons von Independent, der die Ablehnung gesellschaftlicher Konventionen wie Profitstreben, Gehorsam gegenüber Autoritäten oder den politischen Konservatismus der Reagan-Jahre

12 In Sounds wurde ab 1975 eine Auflistung der Album-Nennungen von einem (!) Independent-Plattenladen - d.h. ein vom Eigentümer geführter Laden, der nicht den großen Ketten wie Woolworths, Virgin, HMV oder Tower Records angehört - aufgeführt. Ab 1979 wurden Independent Charts erst im New Musical Express, dann ab 1980 im Melody Maker veröffentlicht. Record Business, das Branchenblatt der englischen Musikindustrie, führte die lange gültige Unterscheidung durch seine 1980 erstmals publizierten Charts ein, wonach die Form des Vertriebs der Platten ausschlaggebend für das Labeling einer Musik als Independent wurde (vgl. Fonarow 2006: 32f.). Diese industriefreundliche Lösung ermöglichte nun den markbeherrschenden Plattenfirmen, ihre Alben ebenfalls in diesen hoch angesehenen Charts listen zu können - sie mussten nur über einen unabhängigen Vertrieb wie etwa The Cartel, eine Unternehmung mehrerer eigentümergeführter Plattenläden, vertrieben werden.

13 Bspw. April - Musik im Vertrieb der Musiker (ab 1976 Schneeball Records) oder für den Jazz FMP (Free Music Production). 
genau so umfasst wie einen bisweilen ans Asketische grenzenden Lebensstil kreativer Selbstverwirklichung. Independent bedeutet zu Beginn der 1980er Jahre, alle Umstände seines Lebens selbst in die Hand zu nehmen und dies als einen politischen Akt der Selbstermächtigung und des Widerstands zu verstehen. "Our band could be your life «, singen die Minutemen (1984) in »History Lesson Pt. II« - Independent ist hier nicht bloß ein musikindustrieller Begriff für die Vermarktung populärer Musik, sondern ein umfassendes Modell der emanzipatorischen Organisation des sozialen Miteinanders.

Heute dagegen, so stellte jüngst eine qualitative Studie fest (Eisewicht/ Grenz 2010), ist Indie eher als Lebensstil junger Menschen zu begreifen, in dem Werte wie Natürlichkeit, Harmonie, Konfliktvermeidung und - tatsächlich! - Anstand auf ein ebenso spießbürgerliches wie opportunistisches Umdeuten von einstmals auch kommerzieller Unabhängigkeit hin zu einem selbstbezüglichen Konsumismus deuten. Als zentrales Prinzip der Sinndeutung und -erzeugung stellen die Autoren ein » Anders-Sein-Wollen « fest, das vor allem in Selbstdarstellung, Kleidung aber auch Verhalten vom - niemals explizit gefassten — »Mainstream« abweicht (ebd.: 96). Eine tatsächlich gelebte Unabhängigkeit, etwa im oben genannten Sinne der Minutemen, sei nicht zu entdecken: "Ganz im Gegenteil zum Postulat der antikommerziellen Unabhängigkeit ist es letztlich sogar eine für die Szenepartizipation notwendige Voraussetzung, eine nicht unerhebliche Konsumptionsbereitschaft mitzubringen« (ebd.: 92f.).

Selbstredend spielen hier Fragen wie die Form des Vertriebs oder die Besitzverhältnisse eines Labels ebenso keine Rolle mehr wie politische oder ethische Implikationen eines unabhängigen Lebens. »Es geht dem selten politisch engagierten Indie nicht um den Sturm der Barrikaden«, führen Eisewicht und Grenz aus, „sondern um eine sozusagen unideologische Selbstbezogenheit, um sich darüber von einem (szene-)umweltlichen Außen abgrenzen zu können« (ebd.: 96). »Indie zu sein« scheint demnach heute vor allem als Differenzbemühung gegenüber anderen Lebensstilangeboten verstanden zu werden. Wenn nun allerdings derart zentrale Mechanismen des Marktes - in einer Welt des Warenüberangebots, in der die nächste Ware sich von der vorhergehenden zu unterscheiden suchen muss, um gekauft zu werden - in die musikalische Praxis des Alltags unhinterfragt übernommen werden, ist es wohl kaum angemessen als »unideologisch« zu bezeichnen. Independent ist stattdessen Ergebnis und Instrument herrschender Ideologie, verstanden im Sinne einer überindividuellen Instanz der internen Legitimierung eines gegebenen gesellschaftlichen Zustands (vgl. Hillmann 2007: 358). 


\section{Independent als ideologische Kategorie und ihre Inszenierung in Musikzeitschriften}

Musikzeitschriften als mediale Inszenatoren entwerfen und proben Strategien zur Aufführung von Independent. Mit Judith Butler (1997: 22) gesprochen: Die performative Inszenierung von Independent erzeugt die Wirkungen, die sie benennt; zu betonen ist, dass diese Hervorbringungen der Macht eines Diskurses unterworfen sind und erst unter ständiger Wiederholung entstehen. Auch die an der Produktion der Musikzeitschrift Beteiligten wissen um die diskursiven Bedeutungen von Independent. Indem sie aber diese Monat für Monat immer wieder >neu < formen und aktualisieren, tragen sie somit zu den beschriebenen Verschiebungen des Begriffs bei. Und Butler weiter folgend, ist es in der Tat so, dass in der Produktion einer Musikzeitschrift diverse Interessen niemals gleichstarker Parteien zusammenlaufen, die für die Untersuchung dieser Hervorbringungen zu berücksichtigen sind. Ich beschränke mich hier auf die besitzenden bzw. leitenden Institutionen und Personen sowie auf diejenigen, die als Medienakteure für die tatsächliche Ausführung der Inszenierung verantwortlich sind, die Journalisten.

Für die Besitzer bzw. die sie vertretenden machtausübenden Institutionen einer Zeitschrift erfüllt Independent zwei Funktionen: Der Begriff bringt ihnen sowohl ökonomisches wie auch symbolisches Kapital. Das liegt daran, dass Medien Inhalte publizieren müssen, die sowohl auf dem Leser- wie auch dem Werbemarkt Nachfrage erzeugen. In der gebotenen Kürze: Kann an einen - unterstellten! - Wertekosmos der Leserschaft thematisch angeschlossen werden, erzeugt man Kaufbereitschaft auf dem Lesermarkt. Kann darauf aufbauend glaubhaft gemacht werden, dass eine bestimmte Leserschaft diese Thematik interessant findet, ${ }^{14}$ ist aufgrund der sich andeutenden Zielgruppengenauigkeit der Zeitschrift der Verkauf einer Anzeige auf dem Werbemarkt wahrscheinlicher geworden und ökonomisches Kapital fließt nun auch aus Werbeerlösen.

Es wäre nun jedoch falsch, daraus eine direkte Abhängigkeit der Inhalte von den Kunden, d.h. die Präsentation von Indie als durch die Werbenden

14 Meist geschieht dies durch eine Gleichsetzung von Titelthema und Absatz des Heftes an den Verkaufsstellen, nicht aber durch eine qualitative Untersuchung der LeserInnen. Quantitative Untersuchungen der Leserschaft, bei denen vor allem Alter, Geschlecht, Bildungsgrad und Haushaltsnettoeinkommen für die bessere Verwertung auf dem Werbemarkt erhoben werden, unternehmen die Verlage nur selten, da sie mit erheblichen Kosten verbunden sind. Im Falle des Rolling Stone etwa vergingen bis zu acht Jahre zwischen der statistischen Erhebung der Leserschaft (vgl. Doehring 2011: 219). 
geleitet zu folgern, denn ein weiterer Aspekt spielt in den Prozess innerhalb dieses »Netzes gegenseitiger Abhängigkeiten« (Doehring 2011: 272) mit hinein: das Image der Musikzeitschrift, das heißt ihr symbolisches Kapital, das wiederum von der Inszenierung des Begriffs Independent profitiert. Am Beispiel der Intro wird dies deutlich: Dieses Magazin wird als indie wahrgenommen, weil es über Indie-Bands (und -filme, -bücher, -klamotten usw.) berichtet, die wiederum als Indie-Bands wahrgenommen werden, weil ein Indie-Magazin darüber schreibt. Zur Erinnerung: Inszenierungen im oben dargestellten Sinn bringen Imaginäres und Fiktives als Empirisches, sprich: Reales hervor. Erst durch die Inszenierung als performativer Akt tritt das Inszenierte in Erscheinung. Intro ist es demnach gelungen, per Inszenierung ihren Status als Indie-Magazin und somit ihre Authentizität symbolisch zu behaupten: Sie gilt als unabhängig, gleichwohl sie aufgrund ihrer Produktionsweise als Gratismagazin höchste Abhängigkeiten eingeht. ${ }^{15}$ Im Gesamten ergibt aus der Inanspruchnahme und Ausdeutung des Begriffs Independent somit ein manifester symbolischer wie materieller Gewinn für den Ort und die Institution dieser monatlichen performativen Äußerungen. ${ }^{16}$

Independent ist jedoch auch ein wichtiger Begriff für die Redakteure, wenn sie planen und entscheiden, was wann und wie im Heft stehen soll. Aufgrund ihrer habituellen Dispositionen - d.h. durch ihre jahrelange intensive Beschäftigung mit populärer Musik, die durch Independent-Bands wenn nicht initiiert, so doch geprägt wurde (vgl. ebd.: 179), und durch das jahrelange Lesen derjenigen Magazine, für die sie nun arbeiten - wissen sie um die diskursiven Bedeutungen und können schnell und effektiv im Rahmen des erforderlichen und zu lernenden beruflichen Handelns Entscheidungen

15 Im Independent-Diskurs der 1980er Jahre, wo gemeinhin Authentizität und Eigenständigkeit als fundamentale Erkennungszeichen galten, wäre diese finanzielle Abhängigkeit als Malus gewertet worden. Diese Werte haben sich zwar, wie gesehen, heute verändert, diesem Umstand scheint Intro jedoch nicht zu trauen. Deshalb ist für sie das Anknüpfen an diesen Diskurs wichtig: Ihr gelingt es im Ganzen, Abhängigkeit mithilfe anderer Inszenierungen von Unabhängigkeit (in Schrift, Bild und Wirken in Internet wie als Konzertveranstalter) vergessen zu machen. »Der Eindruck von Authentizität entsteht gerade als Ergebnis einer besonders sorgfältigen I.[nszenierung]« (Fischer-Lichte 2005a: 149).

$16 \mathrm{Zu}$ berücksichtigen ist hier natürlich der Kampf um die Positionierung des Magazins im - im Bourdieuschen Sinne verstandenen - Feld der Popmusikzeitschriften. Intro festigt ihre Position als Indie-Magazin ebenfalls durch eine visuelle Präsentation, einen betont subjektiven und lockeren journalistischen Tonfall und eine Themenwahl, die alle zusammen auf die Selbstinszenierung als ehemaliges Fanzine - und damit »nah an der Basis « sowie "nicht am Kommerz" orientiert - hindeuten. Die Institution gewinnt somit eine unverwechselbare Kontur in der deutschsprachigen Konkurrenz im Popmusikzeitschriftenmarkt und auch in den Selbstdeutungen der hier Beschäftigten wie Präsentierten (vgl. Doehring 2011: 174ff.). 
fällen. Dies möchte ich am Beispiel von Berichten über die kanadische Band Arcade Fire aus dem Jahr 2010 in der Intro zeigen. ${ }^{17}$

Dort werden in der September-Ausgabe 2010 Arcade Fire in einem Bericht eines freien Mitarbeiters (Happel 2010) beschrieben als eine Band, die dem »Musikbiotop « Montreal entstammt, ${ }^{18}$ einem »sehr schöne[n] Kultur-Ökosystem«, wie der Musiker Matthew Woody von der Montrealer Gruppe Plants and Animals zitiert wird (ebd.: 43). Hier hatten 2005 diverse Musikzeitschriften einen Quell und Hort »indiesker Popmusik« (ebd.) lokalisiert, als Belege werden die Bands Broken Social Scene und Stars genannt. Arcade Fire seien typische Vertreter der Montrealer Szene, da ihnen der Autor »künstlerische Eigenständigkeit« und den »Hang zu Kollaborationen, struktureller Offenheit oder die Weiterentwicklung der eigenen Kunst [sic!]《 (ebd.) attestiert. Ihr Album The Suburbs (2010) - aktualitätsgebundener Anlass der journalistischen Berichterstattung, da zeitnah ${ }^{19}$ zur SeptemberAusgabe der Intro veröffentlicht - behandele die Themen »Altern, die eigene Jugend, Heimat, die Hoffnung auf ein unverfälschtes Leben« (ebd.). Dieses Album, mit »konventionellem Instrumentarium« aufgenommen, verzichte »bewusst « auf Hits, die die beiden vorhergehenden Alben noch enthielten: Das Album The Suburbs »zwingt den Hörer geradezu, es als Ganzes zu begreifen, und es braucht Zeit, um seine Größe zu entfalten. Oder, wie Win Butler [der Sänger von Arcade Fire] es in >We Used To Wait ausdrückt: >l hope something pure can last« (ebd.).

In derselben Ausgabe wird Arcade Fire von der Redaktion die mit »Spektakel « überschriebene umfangreichste Plattenkritik zugestanden. Deren Autor, ein dauerhaft beschäftigter Redakteur, schreibt, dass die Band die ihr zugestandene »musikalische Freiheit« dafür genutzt habe, ein von ihm als »brennend« klassifiziertes Album aufzunehmen - schließlich handele es

17 Analysiert wurde die Berichterstattung in der Intro, der deutschen Ausgabe des Rolling Stone, dem Musikexpress und der Spex; aus Platzgründen konzentriere ich mich auf die Darstellung der Berichterstattung in der Intro.

18 Bei allen im Intro-Artikel bemühten Zuschreibungen an ein organisches Wachstum der Montrealer Musik muss dies allerdings nicht bedeuten, dass die Mitglieder der Band dort geboren und aufgewachsen sein müssen, sondern dass sie lediglich ihren Wohnsitz dort führen: Régine Chassange, die bei Arcade Fire mehrere Instrumente spielt, wurde tatsächlich in Montreal geboren und wuchs in einem Vorort auf, Win Butler dagegen, Sänger und Gitarrist bei Arcade Fire, wurde in Kalifornien geboren und wuchs in Texas auf.

19 Veröffentlichungstermin des Albums war der 30. Juli 2010, die Septemberausgabe der Intro erschien gute zwei Wochen später, am 16. August. 
sich hier um »Kanada-Indie«, der ein hohes Maß an »Enthusiasmus « und »Energie« erwarten lassen dürfe (Steinbrink 2010: 84). ${ }^{20}$

Die von Fonarow benannten Wertmuster sind eindeutig zu identifizieren: Der puristische Aspekt ist im benutzten Instrumentarium zu sehen, Natürlichkeit spielt die Metapher des »Musikbiotops « Montreal genauso eine Rolle wie die künstlerische Eigenständigkeit und musikalische Freiheit als Autonomiebestrebungen gedeutet werden dürfen, die außerdem beide auf den Geniekult und das hohe Ansehen des kreativen Ausdrucks als Erben des Romantizismus verweisen. Die damit verbundene Leidenschaft wird als enthusiastisch gekennzeichnet, Nostalgie und moralischer Rigorismus sind in der Hoffnung auf das bessere, unverfälschte Leben von früher in den Vororten präsent. Gleich mehrfach wird der Begriff Indie zur Kategorisierung dieser Musik und der Musiker bemüht.

In derselben Ausgabe der Intro ist lediglich eine Sechstelseite Werbung abgedruckt, in der für Platte und Tour (mit drei Stationen) geworben wird (Intro 185: 87). Eine direkte Verknüpfung von Anzeigenschaltung und Berichterstattungsanlass - wie oft im Zusammenhang mit musikjournalistischer Berichterstattung gemutmaßt - scheint aufgrund des geringen Umfangs dieser Anzeige und somit der eingenommenen Werbekosten zunächst schnell ausgeschlossen werden zu können. Doch muss man wissen, dass Themen in Absprachen zwischen Redaktion und »Medienpartnern « geplant werden, die z.T. mehrere Monate vor der eigentlichen Veröffentlichung liegen und ihre Wirkungen möglichst über einen längeren Zeitraum entwickeln sollen. Denn schließlich muss Intro ebenso wie ihre hier beschäftigten Medienakteure auf dem Feld des Popmusikjournalismus um ihre jeweilige Position kämpfen. Daher lohnt sich der Blick auf die folgenden Ausgaben: The Suburbs wird in der Oktober-Ausgabe sowohl von den Lesern (Intro 186: 79) als auch im so genannten »Platten vor Gericht«-Mehrjurorentest (ebd.: 80f.) zur >besten< Platte der Ausgabe gewählt; die zehnteilige Jury setzt sich zu vier Fünfteln aus Musikern zusammen, ein Platz wird an Leser bzw. User von intro.de vergeben, einer ist einem Intro-Mitarbeiter vorbehalten, der in diesem Fall übrigens wiederum Christian Steinbrink ist - zur Erinnerung: Redakteur der Intro und Rezensent des Albums in Ausgabe 185. The Suburbs führt in den Leser-Charts auch der November-Ausgabe (Intro 187: 77). In diesem Heft wird außerdem, zwei Monate nach den ersten Arcade Fire-

20 Nur kurz der Blick auf die anderen Musikzeitschriften: Auch im Rolling Stone (Brüggemeyer 2010) wird vierseitig über die Band berichtet, im ebenfalls bei Axel Springer erscheinenden Musikexpress ziert die Band das Cover und erhält ebenfalls eine mehrseitige Berichterstattung, in der Spex (Hammelehle 2010) wird zeitgleich über mehrere Seiten berichtet. Die Wertmuster für Independent lassen sich auch hier belegen. 
Berichten, das für den europäischen Vertrieb von Arcade Fire zuständige Label City Slang auf vier Seiten »gefeatured«, wie es im Jargon heißt (Venker et al. 2010). Berichterstattungsanlass ist das 20-jährige Bestehen des Labels. ${ }^{21}$ Das bedeutet konkret, dass Labelbetreiber Christof Ellinghaus dem Intro-Chefredakteur Thomas Venker aus seinen Erinnerungen erzählen darf und elf Intro-Mitarbeiter die sbesten< Platten sbesprechen<, die bei City Slang erschienen sind - fast die Hälfte der Musikjournalisten, die auf den die Texte begleitenden Fotos abgebildet sind, halten die Vinyl- oder Kassetten-Ausgabe des jeweils rezensierten Albums in der Hand, Symbole der von Fonarow als Indie-typisch geltenden Kultivierung nostalgischer Technologien.

Die ebenfalls bei City Slang (übrigens in Großbritannien gemeinsam mit V2 Records - und d.h. Universal, vgl. Discogs 2012a) unter Vertrag stehende Band Caribou erhält in derselben Ausgabe die dem City Slang-Bericht vorhergehenden vier Seiten (Raffeiner 2010), auf denen Sänger Dan Snaith per Zitat das Label noch einmal ausdrücklich lobend hervorhebt. Snaith ist außerdem auf dem Cover der Ausgabe zu sehen, ${ }^{22}$ im Editorial (Intro-Redaktion 2010: 3) wird ihm, diesem so inszenierten »Wider-Willen-Popstar «, der Titel einer »neue[n] Ikone der >Indie-Kirche« verliehen. City Slang schaltet in dieser Ausgabe (Intro 187: 85) nun eine halbe Seite für Caribous Album Swim (2010) und die zugehörige Tour (mit Sponsoring durch Electronic Beats, dem »international music program« der PR-Abteilung der Deutschen Telekom AG). Die andere halbe Seite belegt das Label Souterrain Transmissions, an dessen Gründung Christof Ellinghaus von City Slang beteiligt war (vgl. »Maximilian« 2010) und das im selben Gebäude wie City Slang beheimatet ist.

Zusammengefasst: Natürlich spielen für die Präsentation Arcade Fires in der Intro finanzielle Aspekte eine Rolle. Es greift jedoch zu kurz, will man diesen allein den Berichterstattungsanlass und die dann gewählte Darstellung zuschreiben, denn zum einen wird Popmusikjournalismus in einem Netz gegenseitiger Abhängigkeiten hergestellt. Sowohl City Slang (und damit davon ist wenigstens auszugehen - die Musiker), die Deutsche Telekom AG als auch die Intro profitieren von dieser »Kooperation« zwischen »Medien-

21 City Slang existiert strenggenommen zwar erst seit 1992 eigenständig, da es zuvor ein Sublabel von Vielklang war, aus s guten G Gründen will dies wohl niemand bemerken. Einen Berichterstattungsanlass wie diesen ließ sich bspw. auch schon zum angeblich 10-jährigen Jubiläum Thomas Bohnet (2000) nicht entgehen.

22 Auf dem Cover wird höchst subtil die Zusammengehörigkeit von Snaith und City Slang illustriert: Die Farben des Anoraks des Musikers werden im Banner, das auf den Bericht über City Slang hinweist, wieder aufgenommen. 
partnern «, wie es im Feld genannt wird, ohne die es wohl auch einmal geht, mit der aber alle gut leben können. Zum anderen muss das Handeln der Medienakteure bedacht werden, die sich mit diesem Umfeld der Berichterstattung arrangieren müssen; umso besser, wenn es wie bei Arcade Fire und Caribou um >richtig gute - Indie-Musik geht, denn diese können sie als Musikexperten - so lässt sich zumindest ihr Selbstbild zusammenfassen (vgl. Doehring 2011: 176) - aufgrund ihrer habituell erworbenen Musikkonzepte beurteilen und genießen.

Es lässt sich hier zeigen, dass Independent als Begriff eines Diskurses nicht nur die beschriebenen Funktionen für die Magazine und Journalisten erfüllt, sondern zudem die oben vorgestellte ideologische Funktion einnimmt: In dem Moment, wo auf Independent Bezug genommen wird, können Widersprüche verdeckt und neue Bedeutungsallianzen etabliert werden, die uns dann in den dargelegten Beschreibungen der heutigen Indies gegenübertreten. Wenn etwa der Autor des Arcade Fire-Artikels die Entwicklung der hochgelobten Montrealer Musikszene auf die »kluge staatliche Kulturarbeit « (Happel 2010: 43) der kanadischen Regierung zurückführt, wie sie sich in der Arbeit von FACTOR (The Foundation Assisting Canadian Talent On Recording) ${ }^{23}$ darstellt, aber den Zusammenhang von Förderung und Abhängigkeit

23 FACTOR ist eine private Stiftung, die Fördergelder privater Radiostationen und des Canada Music Found, angesiedelt im Department of Canadian Heritage, verteilt. Zum Ziel hat sie die Förderung der kanadischen Musikindustrie - und nicht etwa der kanadischen Musikkultur insgesamt. Im Steuerjahr 2010-2011 hat FACTOR insgesamt 15,9 Millionen Kanadische Dollar für die Förderung der »Canadian independent music industry « ausgegeben (vgl. FACTOR 2011). Wie autonom eine in diesem Ausmaß geförderte independent music scene (zur Erinnerung: unabhängige Musikszene) tatsächlich ist, müsste erörtert werden. Fest steht, dass staatliche Intervention unser Bild von »Kanada-Indie«, wie die Intro schreibt, wesentlich geprägt hat. Zum Vergleich: Nicht bloß ein bundesdeutsches »Ministerium für das deutsche Erbe« wäre - noch - unvorstellbar, sondern auch die Höhe der Förderung und deren Ausrichtung auf Independent ist nicht vergleichbar: Seit Ende 2007 gibt es die Initiative Musik Projektgesellschaft $\mathrm{GmbH}$, eine »Fördereinrichtung der Bundesregierung für die Musikwirtschaft in Deutschland «, an der der Bundesbeauftragte für Kultur und Medien (Staatsminister Bernd Neumann), die GVL, die GEMA und der Deutsche Musikrat beteiligt sind. Die Fördergelder von etwa 2,3 Millionen Euro werden zum überwiegenden Teil vom Bundesbeauftragten getragen (2010 etwa 2 Millionen Euro), 180.000 Euro kommen je von GVL und GEMA (vgl. Initiative Musik 2011). Angesichts einer laut des Internationalen Währungsfonds im Jahr 2011 etwa doppelt so hohen Wirtschaftskraft Deutschlands im Vergleich zu Kanada (BIP in Mio. USDollar: Deutschland 3.577.031, Kanada 1.736.869; vgl. International Monetary Fund 2012), ist dieser Betrag als marginal zu bezeichnen. Und zur besseren Einordnung des Stellenwertes bundesdeutscher Förderkultur populärer Musik abschließend ein Blick auf die hiesige Filmförderung, die vom Staatsminister mit 90 Millionen Euro jährlich unterstützt wird (vgl. Presse- und Informationsamt der Bundesregierung 2011). 
nicht reflektiert; wenn etwa in allen Magazinen der Hinweis auf das für den US-Markt zuständige Indie-Label Merge Records erfolgt, ohne dass auf den Vertrieb durch die sich zwar indie gebende ADA (Alternative Distribution Alliance) geachtet wird, die jedoch tatsächlich ein Joint Venture von Sub Pop und Warner im Verhältnis von 5 zu 95 Prozent Anteilen ist (vgl. auch Oakes 2009: 150); wenn niemandem auffällt (weil man es nicht fragt!), dass das für den europaweiten mp3-Vertrieb zuständige Label Mercury eine Tochter von Universal Music ist; wenn man sich nicht wundert, warum Universal auch noch den Vertrieb und Verlag für einen ganzen Subkontinent (Südamerika) übernimmt (vgl. Discogs 2012b) - dann ist Independent ein höchst geeigneter Begriff, den Prozess der Warenproduktion und des Warenvertriebs nicht nur zu verdecken, sondern auch noch mit symbolischer Bedeutung zu versehen und somit in neue Kontexte überführen zu können.

Im Ergebnis ereignet sich hier die symbolische Legitimierung kommerziellen Eingreifens in Musikprozesse, wie es Wesen des gegenwärtigen Musikgeschäfts ist - und ob von staatlicher oder privatwirtschaftlicher Hand kommerziell eingegriffen wird, scheint dann letztlich auch egal zu sein, denn unabhängig ist hier nichts und niemand. Und wohlgemerkt: Die ideologische Funktion der Inszenierung von Independent funktioniert gerade deshalb, weil die Musikjournalisten sie nicht erkennen - können! Die Strukturen der Arbeit im Popmusikjournalismus äußern sich in der Etablierung eines für den gegenwärtigen Kapitalismus typischen und notwendigen Berufsethos: Die Musikjournalisten verstehen sich selbst als kreative und freie, d.h. selbstständig beschäftigte sowie durch überwiegend interne Kontrollinstanzen motivierte Arbeiter, die in unterbezahlter, überlanger und weitgehend unhinterfragter Selbstausbeutung ihre Talente und ihr Wissen einem Markt zur Verfügung stellen, der sie für die symbolische Aufladung von Musikwaren und anderen Lifestyleprodukten benutzt. Die Strukturen und Kräfte des Feldes Popmusikjournalismus erzeugen und verlangen diesen intern verankerten Berufshabitus, der bei Bourdieu (1998: 25) als »Brille« die Wirklichkeitswahrnehmung beeinflusst. Kritik ist hier nicht mehr vorgesehen.

Doch halt! Hat nicht die Spex im selben Monat einen Artikel (Hammelehle 2010) publiziert, der sich kritisch mit der Inszenierung von Arcade Fire auseinandersetzt? Dort wird in der Tat die Selbstdarstellung der Band als janusköpfig ausgelegt: Obwohl Arcade Fire sich dezidiert und somit den genannten Wertmustern entsprechend den üblichen Bewerbungsprozessen ihres Albums verweigern, erkennt der Autor Aktivitäten der Band, die diesem Ideal widersprächen. Bezogen wird dies auf ein Pariser Konzert Arcade Fires, welches zugleich zu Ehren eines gestorbenen »Sound-Ingenieurs « und 
als Promotion-Konzert für das neue Album gedient habe. Das Verweigern von Promotion-Aktivitäten (etwa das Anbieten und Wahrnehmen von Presseterminen) wie Promotion-Gegenständen (Gratis-CDs, Informationsbroschüren, Promotion-Videos usw. für die Journalisten) durch Label und Band einerseits werde andererseits durch die Veröffentlichung von Videos im Internet und Vinyl-Singles in ausgesuchten kleinen Plattenläden unterlaufen. Natürlich klingt hier auch gekränktes Gewohnheitsrecht des Musikjournalisten durch. Doch wird nun dieser an sich Indie-typische Verweigerungsmodus zur Inszenierung von Independent erklärt: »Die Inszenierung ist bei Arcade Fire eben immer ebenso wichtig wie die Musik, gerade weil es die Inszenierung offiziell gar nicht gibt « (Hammelehle 2010: 23). Diese »Inszenierung musikalischer Blockbuster«, garniert gar mit einem »Deal« mit American Express über 1,5 Millionen Dollar (ebd.), widerspreche dem Independent-Diskurs zutiefst. So wird denn auch befürchtet, dass »Stadionrocker[n]« wie den für die Vielen musizierenden U2 oder Bruce Springsteen die »Indie-Kanonisierung durch die Hintertür« (ebd.) gelingen könne: Da Arcade Fire ähnliche Musik wie diese spielten, sich ähnlich inszenierten und dabei trotzdem als Independent-Band gälten, befürchtet der Spex-Autor ein Übergreifen des Independent-Begriffs in Bereiche, in denen er ihm zufolge nichts verloren habe.

Die Spex-Redakteure greifen daher für die Präsentation der Band im Heft zu rabiaten Mitteln, denn Spex versteht und inszeniert sich eine als Zeitschrift, die sich vorgenommen hat, alle im Heft gedruckten Fotos selber zu machen (vgl. Dax 2008: 13) - aus Gründen einer postulierten Unabhängigkeit, wohl aber auch, um sich mindestens visuell von der Konkurrenz der anderen Musikzeitschriften zu unterscheiden. Da Arcade Fire für keine im Feld etablierten und erwarteten Promotion-Aktivitäten zur Verfügung standen, wo ein von der Spex erwünschtes Foto hätte geschossen werden können, druckt das Magazin nun zum Artikel die von deren Label City Slang bereitgestellten Fotos - in betont schlechter Graustufenauflösung, über die in grüner gefetteter Blockschrift »THIS IS A PROMOTIONAL IMAGE« geschrieben wurde.

Ist dies nun die geforderte Kritik aus den Reihen der Popmusikjournalisten? Meines Erachtens nein. Denn hier wurde eine Chance vergeben: Anstatt in diesem Artikel den Warencharakter der Musik und die Bedeutung und somit das Funktionieren des ideologischen Modells Independent auch für die Spex eindeutig zu benennen, geriert sich die Spex, d.h. gerieren sich die hier tätigen Medienakteure vom freien Mitarbeiter bis zur Redaktion, als Hüter des >wahren< Independent - und tragen so zum Fortbestehen dieses Begriffs bei. Und dies übrigens umfangreich auf vier Seiten, denn einen 
Bericht über Arcade Fire, dieses »interessante Thema « (wie es im Jargon heißt), kann die oben genannte Logik der Produktion nicht ignorieren - den muss man bringen. Übrigens: Die Leser bestätigen dieses strategische Inszenieren im Nachhinein, denn in den Jahresendlisten rangiert The Suburbs in der Gunst als beliebtes Indie-Album des Jahres weit oben - auch in der Spex. ${ }^{24}$

Es ist natürlich davon auszugehen, dass der Diskurs über Independent beim Musikmagazin weder beginnt noch endet, sondern dass dieser Begriff bereits viel eher in das Handeln der professionellen (und wahrscheinlich bereits semiprofessionellen) Akteure erster (Musiker) und zweiter (Plattenfirma) Instanz eingegangen ist. Nicht bloß die Planung, Aufbereitung und Präsentation von Medieninszenierungen wäre daher zu untersuchen, sondern die ihnen vorausgehenden Planungs- und Entscheidungsprozesse der musikalischen sowie die Auswahl- und Gestaltungsbedingungen musikindustrieller Akteure, in die bereits Überlegungen bezüglich der Möglichkeit medialer Inszenierungsstrategien ihren Einfluss nehmen dürften. Oder wie es einer der Musikredakteure ausdrückte: »Und die Bands sollen dann natürlich auch funktionieren, deshalb sehen sie auch schon so aus, wie sie glauben, dass sie dann durchkommen durch den Strom « (IN 1: 6, in Doehring 2011: 269). Dazu bedarf es unbedingt weiterer Untersuchungen. Denn Independent war, ist und - sehr wahrscheinlich - bleibt ein wichtiger Begriff der Organisation des popmusikalischen Lebens, mit dessen Hilfe die Etablierung der Camouflage höchst abhängiger Inszenierungen von Unabhängigkeit in der musikalischen Praxis bestens gelingt.

24 Bei den Spex-Lesern ist am Ende des Jahres The Suburbs auf Platz 2 hinter Caribous Swim zu finden, in den Spex-Redaktionscharts liegt The Suburbs auf Rang 11, Swim auf dem dritten Rang (Spex 2011: 58 u. 62). Ein kurzer Blick in die Intro-Jahrescharts, die sinnigerweise mit »Tag der Abrechnung « überschrieben sind: Die Redaktion wählte The Suburbs auf den zweiten, Swim auf den ersten Platz der sbesten Alben des Jahres, die Leser der Intro drehten das Verhältnis um: The Suburbs ist das hier erstplatzierte, Swim das dahinter auf Platz 2 liegende Album des Jahres 2010 (Intro 2011: 12f.). 


\section{Literatur}

Alsmann, Götz (1985). Nichts als Krach. Die unabhängigen Schallplattenfirmen und die Entwicklung der amerikanischen populären Musik 1943-1963. Drensteinfurt: Huba.

Anon. (2012). »Artic Monkeys.« In: Wikipedia.org, http://de.wikipedia.org/wiki/ Arctic_Monkeys (Stand: 9.9.2012, 13:47 Uhr; Zugriff: 17.9.2012).

Azzerad, Michael (2002). Our Band Could Be Your Life. Scenes from the American Indie Underground 1981-1991. New York: Back Bay Books.

Bannister, Matthew (2006). White Boys, White Noise. Masculinities and 1980s Indie Guitar Rock. Aldershot, Burlington: Ashgate.

Blaukopf, Kurt (1984). Musik im Wandel der Gesellschaft. Grundzüge der Musik soziologie. München, Kassel u. a.: dtv / Bärenreiter.

Blaukopf, Kurt (2010). »Musikpraxis als Gegenstand der Soziologie.«In: Ders. Was ist Musiksoziologie? Ausgewählte Texte. Hg. v. Michael Parzer (= Musik und Gesellschaft 28). Frankfurt/M. u. a.: Lang, S. 89-106.

Bohnet, Thomas (2000). »Wenn mit dem Scheckbuch gewedelt wird. Das Berliner Independent-Label City Slang wird zehn Jahre alt.« In: Leeson, Nr. 12, http:// www.inselmedia.de/leeson/nr12/label_city_slang.htm (Stand: 28.12.2001; Zugriff: 13.9.2012).

Bourdieu, Pierre (1998). Über das Fernsehen. Frankfurt/M.: Suhrkamp.

Broven, John (2009). Record Makers and Breakers. Voices of the Independent Rock ' $n$ ' Roll Pioneers. Urbana / Chicago: University of Illinois Press.

Brüggemeyer, Maik (2010). »Arcade Fire. Vorstadtkrokodile auf Heimaturlaub.«In: Rolling Stone (dt. Ausg.), H. 190 (August), S. 48-51.

Büsser, Martin (1997). "Stereolab. Zur Konstruktion von Indie-Stars. In: Kulturindustrie. Kompaktes Wissen für den Dancefloor. Hg. v. Martin Büsser et al. (= Testcard - Beiträge zur Popgeschichte 5). Mainz: Ventil, S. 108-115.

Butler, Judith (1997). Körper von Gewicht. Die diskursiven Grenzen des Geschlechts. Frankfurt/M.: Suhrkamp.

Clawson, Mary Ann (1999). »When women play the bass: Instrument spezialization and gender interpretation in alternative rock music. " In: Gender \& Society 13, Nr. 2, S. 193-210.

Davis, Helen (2001). "All Rock and Roll Is Homosocial: The Representation of Women in the British Rock Music Press. "In: Popular Music 20, Nr. 3, S. 301-319.

Dax, Max (2008). »Editorial.«In: Spex, H. 314 (Mai/Juni), S. 13.

Discogs (2012a). »Caribou. Swim.« In: discogs.com, http://www.discogs.com/ Caribou-Swim/master/240989 (Zugriff: 17.9.2012).

Discogs (2012b). »Arcade Fire. The Suburbs. «In: discogs.com, http://www.discogs. com/Arcade-Fire-The-Suburbs/master/264742 (Zugriff: 17.9.2012).

Doehring, André (2011). Musikkommunikatoren. Berufsrollen, Organisationsstrukturen und Handlungsspielräume im Popmusikjournalismus (= texte zur populären musik 7). Bielefeld: transcript.

Eisewicht, Paul / Grenz, Tilo (2010). „Frei und auf den Beinen und gefangen will ich sein. Über die »Indies«. Berlin: Archiv der Jugendkulturen.

FACTOR (2011). »Fostering Emerging Artists and Musical Diversity. Annual Report 2010-2011.« Online unter: http://www.factor.ca/docs/AnnualReports/FACTOR_ Annual_Report_2010-2011.pdf (Zugriff: 9.9.2012). 
Fischer-Lichte, Erika (1998). »Inszenierung und Theatralität. In: Inszenierungsgesellschaft. Ein einführendes Handbuch. Hg. v. Herbert Willems und Martina Jurga. Opladen: Westdeutscher Verlag, S. 81-90.

Fischer-Lichte, Erika (2002). »Grenzgänge und Tauschhandel. Auf dem Weg zu einer performativen Kultur.«In: Performanz. Zwischen Sprachphilosophie und Kulturwissenschaften. Hg. v. Uwe Wirth. Frankfurt/M.: Suhrkamp, S. 277-300.

Fischer-Lichte, Erika (2005a). »Inszenierung. «In: Metzler Lexikon Theatertheorie. Hg. v. Erika Fischer-Lichte, Doris Kolesch und Matthias Wartstatt. Stuttgart, Weimar: Metzler, S. 146-153.

Fischer-Lichte, Erika (2005b). »Performativität/performativ.«In: Metzler Lexikon Theatertheorie. Hg. v. Erika Fischer-Lichte, Doris Kolesch und Matthias Wartstatt. Stuttgart, Weimar: Metzler, S. 234-242.

Fonarow, Wendy (2006). Empire of Dirt. The Aesthetics and Rituals of British Indie Music. Middletown: Wesleyan University Press.

Foucault, Michel (1991). Die Ordnung des Diskurses. Mit einem Essay von Ralf Konersmann. Frankfurt/M.: Fischer.

Goffman, Erving (1983). Wir alle spielen Theater. Die Selbstdarstellung im Alltag. München: Piper.

Gruber, Siegfried (1995). Das Konsumentenverhalten bei Independent-Tonträgern. Eine empirische Untersuchung der Käuferschaft von »unpopulärer Populärmusik« unter besonderer Berücksichtigung methodischer Erkenntnisinteressen. Frankfurt/M. u. a.: Lang.

Gukenbiehl, Hermann L. (2000). »Institution und Organisation.«In: Einführung in Hauptbegriffe der Soziologie. Hg. v. Hermann Korte und Bernhard Schäfers. Opladen: Leske u. Budrich (5., erw. u. akt. Aufl.), S. 141-157.

Hammelehle, Sebastian (2010). „Arcade Fire. Entscheidende Schritte in Richtung Epos. «In: Spex, H. 328 (September/Oktober), S. 20-23.

Happel, Lutz (2010). »Arcade Fire / Stars / Plants and Animals. Die Unberechenbaren gehen baden. «In: Intro, H. 185 (September), S. 42-44.

Hempfer, Klaus W. / Volbers, Jörg (2011). »Vorwort. «In: Theorien des Performativen. Sprache - Wissen - Praxis. Eine kritische Bestandsaufnahme. Hg. v. dens. (= Edition Kulturwissenschaft 6). Bielefeld: transcript, S. 7-12.

Hillmann, Karl-Heinz (2007). Wörterbuch der Soziologie. Stuttgart: Alfred Kröner (5., vollst. überarb. u. erw. Aufl.).

Initiative Musik (2011). »Zielsetzung. « In: initiative-musik.de, http://www. initiative-musik.de/index.php?id=27 (Zugriff: 9.9.2012).

International Monetary Fund (2012). »World economic outlook database April 2012.« In: imf.org, http://www.imf.org/external/pubs/ft/weo/2012/01/ weodata/index.aspx (Zugriff: 18.9.2012).

Intro (2011). »Poll 2010. Der Tag der Abrechnung. «In: Intro, H. 189 (Februar), S. 12-15.

»Maximilian« (2010). "Souterrain Transmissions. Interview mit Krista Schmidt.« In: roteraupe.de, http://www.roteraupe.de/interview/119/souterrain-transmission s.html (Stand: 15.5.2010; Zugriff: 8.9.2012).

Moore, Ryan (2010). Sells Like Teen Spirit. Music, Youth Culture, And Social Crisis. New York, London: New York University Press.

Oakes, Kaya (2009). Slanted and Enchanted. The Evolution of Indie Culture. New York: Holt.

Oldag, Andreas (2012). »EMI am Ende.«In: Süddeutsche Zeitung, Nr. 220, 22./23. September (Samstag/Sonntag), S. 28. 
Oxford Dictionaries (2012). »Indie." In: oxforddictionaries.com, http://oxford dictionaries.com/definition/english/indie?region=us (Zugriff: 14.8.2012).

Presse- und Informationsamt der Bundesregierung (2011). »Filmförderung. «In: bundesregierung.de, http://www.bundesregierung.de/Webs/Breg/DE/Bundesregier ung/BeauftragterfuerKulturundMedien/medien/filmfoerderung/_node.html;jses sionid=A835A94FA92DD5BB8729B3AC5CC61084.s2t1 (Zugriff: 9.9.2012).

Raffeiner, Arno (2010). »Caribou. Im Kleinbus mit dem Nerd des Jahres. «In: Intro, H. 187 (November), S. 26-29.

Spex (2011). »Jahrescharts 2010. «In: Spex, H. 330 (Januar/Februar), S. 58-63.

Steinbrink, Christian (2010). »Arcade Fire. ’The Suburbs<.«In: Intro, H. 185 (September), S. 84.

Umathum, Sandra (2005). »Performance.«In: Metzler Lexikon Theatertheorie. Hg. v. Erika Fischer-Lichte, Doris Kolesch und Matthias Wartstatt. Stuttgart, Weimar: Metzler, S. 231-234.

Venker, Thomas et al. (2010). »20 Jahre City Slang. Gimme Indierock!« In: Intro, H. 187 (November), S. 30-33.

Wartstat, Matthias (2005). »Theatralität.«In: Metzler Lexikon Theatertheorie. Hg. v. Erika Fischer-Lichte, Doris Kolesch und Matthias Wartstatt. Stuttgart, Weimar: Metzler, S. 358-364.

Wicke, Peter (2004). "Soundtracks. Popmusik und Pop-Diskurs.«In: Was ist Pop? Zehn Versuche. Hg. v. Walter Grasskamp, Michaela Krützen und Stephan Schmitt. Frankfurt/M.: Fischer, S. 115-139.

Wicke, Peter / Ziegenrücker, Wieland / Ziegenrücker, Kai-Erik (2007). Handbuch der populären Musik. Geschichte. Stile. Praxis. Industrie. Mainz: Schott (erw. Neuausgabe).

Wiene, Dieter (2012). »Dntel. Aimlessness [Kritik].«In: Groove, Nr. 136 (Mai/Juni), S. 89.

Willems, Herbert (1998). »Inszenierungsgesellschaft? Zum Theater als Modell, zur Theatralität von Praxis. «In: Inszenierungsgesellschaft. Ein einführendes Handbuch. Hg. v. Herbert Willems und Martina Jurga. Opladen: Westdeutscher Verlag, S. 23-80.

Willems, Herbert / Jurga, Martin (1998a). »Einleitung: Zum Aufbau des Buches und zu den Beiträgen. «In: Inszenierungsgesellschaft. Ein einführendes Handbuch. Hg. v. dens. Opladen: Westdeutscher Verlag, S. 9-21.

Willems, Herbert / Jurga, Martin (Hg.) (1998b). Inszenierungsgesellschaft. Ein einführendes Handbuch. Opladen: Westdeutscher Verlag.

Wirth, Uwe (2002). »Der Performanzbegriff im Spannungsfeld von Illokution, Iteration und Indexikalität. «In: Performanz. Zwischen Sprachphilosophie und Kulturwissenschaften. Hg. v. dems. Frankfurt/M.: Suhrkamp, S. 9-60.

\section{Diskografie}

Arcade Fire (2010). The Suburbs. City Slang 2742629.

Caribou (2010). Swim. City Slang SLANG9550054.

Minutemen (1984). »History Lesson Pt. II.«Auf: Double Nickles On The Dime, SST 28. 


\begin{abstract}
Independent is a discourse about popular music that strategically positions popular music's practices from production to distribution and reception. As a historic term, the meaning of independent is never stable; several changes of its connotations are presented. As discourses are led by institutions like popular music magazines, this article shows how and why these magazines use and thereby shape our notion of independent music. For the magazines and its music journalists, independent music serves to acquire economic as well as symbolic capital. But on the whole, it is argued, independent serves as an ideological term camouflaging popular music's capitalist fundamentals. A close reading of 2010's media coverage of Arcade Fire and Caribou is given.
\end{abstract}

